\title{
Factorization, resummation and sum rules for heavy-to-light form factors
}

\author{
Yu-Ming Wang ${ }^{1,2, \star}$ \\ ${ }^{1}$ Fakultät für Physik, Universität Wien, Boltzmanngasse 5, 1090 Vienna, Austria \\ ${ }^{2}$ School of Physics, Nankai University, 300071 Tianjin, China
}

\begin{abstract}
Precision calculations of heavy-to-light form factors are essential to sharpen our understanding towards the strong interaction dynamics of the heavy-quark system and to shed light on a coherent solution of flavor anomalies. We briefly review factorization properties of heavy-to-light form factors in the framework of QCD factorization in the heavy quark limit and discuss the recent progress on the QCD calculation of $B \rightarrow \pi$ form factors from the light-cone sum rules with the $B$-meson distribution amplitudes. Demonstration of QCD factorization for the vacuum-to- $B$-meson correlation function used in the sum-rule construction and resummation of large logarithms in the short-distance functions entering the factorization theorem are presented in detail. Phenomenological implications of the newly derived sum rules for $B \rightarrow \pi$ form factors are further addressed with a particular attention to the extraction of the CKM matrix element $\left|V_{u b}\right|$.
\end{abstract}

\section{Introduction}

Heavy-to-light form factors serve as fundamental inputs of describing many exclusive heavy hadron decays which are of great phenomenological interest to the ongoing and forthcoming collider experiments. Extensive efforts have been devoted to develop systematic theoretical frameworks for the precision calculation of heavy-to-light form factors in QCD (see, for instance[1-7]). In addition to the nonperturbative hadronic distribution amplitudes (DA), the symmetry-conserving "soft" form factors have to be introduced in QCD factorization for $B \rightarrow \pi$ form factors due to the emergence of rapidity divergences in the corresponding convolution integrals. An alternative approach to compute $B \rightarrow \pi$ form factors is QCD light-cone sum rules (LCSR) constructed from the vacuum-to- $B$-meson correlation functions with the aid of the dispersion relations and parton-hadron duality approximation [8-11]. In the following, we will first establish QCD factorization for the vacuum-to- $B$-meson correlation function at one loop employing the method of regions and perform the resummation of large logarithms in the hard and jet functions with the renormalization-group approach in section 2, where the resummation improved LCSR for $B \rightarrow \pi$ form factors and a new determination of the CKM matrix element $\left|V_{u b}\right|$ are also presented.

\footnotetext{
^e-mail: yu-ming.wang@univie.ac.at
} 


\section{The LCSR for the $B \rightarrow \pi$ form factors at $O\left(\alpha_{s}\right)$}

The construction of LCSR for the $B \rightarrow \pi$ form factors can be achieved with the following correlation function [12]:

$$
\begin{aligned}
\Pi_{\mu}(n \cdot p, \bar{n} \cdot p) & =\int d^{4} x e^{i p \cdot x}\left\langle 0\left|T\left\{\bar{d}(x) h \gamma_{5} u(x), \bar{u}(0) \gamma_{\mu} b(0)\right\}\right| \bar{B}(p+q)\right\rangle \\
& =\Pi(n \cdot p, \bar{n} \cdot p) n_{\mu}+\widetilde{\Pi}(n \cdot p, \bar{n} \cdot p) \bar{n}_{\mu},
\end{aligned}
$$

where $p+q=m_{B} v$ and the two light-cone vectors satisfy the relations $n \cdot v=\bar{n} \cdot v=1$. To facilitate the perturbative calculation of short-distance functions in the factorization formula of (1), we need to establish the power counting scheme for the external momentum $p_{\mu}$

$$
n \cdot p \simeq \frac{m_{B}^{2}+m_{\pi}^{2}-q^{2}}{m_{B}}=2 E_{\pi}, \quad \bar{n} \cdot p \sim O\left(\Lambda_{\mathrm{QCD}}\right)
$$

Applying the light-cone operator-produce-expansion (OPE) at space-like $p^{2}$ yields the tree-level factorization formula

$$
\begin{aligned}
& \widetilde{\Pi}(n \cdot p, \bar{n} \cdot p)=\tilde{f}_{B}(\mu) m_{B} \int_{0}^{\infty} d \omega^{\prime} \frac{\phi_{B}^{-}\left(\omega^{\prime}\right)}{\omega^{\prime}-\bar{n} \cdot p-i 0}+O\left(\alpha_{s}\right), \\
& \Pi(n \cdot p, \bar{n} \cdot p)=O\left(\alpha_{s}\right) .
\end{aligned}
$$

in the heavy quark limit, where the $B$-meson light-cone DA in the coordinate space is defined as

$$
\left\langle 0\left|\bar{d}_{\beta}(\tau \bar{n})[\tau \bar{n}, 0] b_{\alpha}(0)\right| \bar{B}(p+q)\right\rangle=-\frac{i \tilde{f}_{B}(\mu) m_{B}}{4}\left\{\frac{1+\hbar}{2}\left[2 \tilde{\phi}_{B}^{+}(\tau)+\left(\tilde{\phi}_{B}^{-}(\tau)-\tilde{\phi}_{B}^{+}(\tau)\right) / n\right] \gamma_{5}\right\}_{\alpha \beta} .
$$

Applying the standard definitions of $B \rightarrow \pi$ form factors and the pion decay constant

$$
\begin{aligned}
\left\langle\pi(p)\left|\bar{u} \gamma_{\mu} b\right| \bar{B}\left(p_{B}\right)\right\rangle & =f_{B \pi}^{+}\left(q^{2}\right)\left[p_{B}+p-\frac{m_{B}^{2}-m_{\pi}^{2}}{q^{2}} q\right]_{\mu}+f_{B \pi}^{0}\left(q^{2}\right) \frac{m_{B}^{2}-m_{\pi}^{2}}{q^{2}} q_{\mu}, \\
\left\langle\pi(p)\left|\bar{d} / n \gamma_{5} u\right| 0\right\rangle & =-i n \cdot p f_{\pi},
\end{aligned}
$$

it is straightforward to derive the hadronic dispersion relation for the correlator (1)

$$
\begin{aligned}
& \Pi_{\mu}(n \cdot p, \bar{n} \cdot p) \\
& =\frac{f_{\pi} n \cdot p m_{B}}{2\left(m_{\pi}^{2}-p^{2}\right)}\left\{\bar{n}_{\mu}\left[\frac{n \cdot p}{m_{B}} f_{B \pi}^{+}\left(q^{2}\right)+f_{B \pi}^{0}\left(q^{2}\right)\right]+n_{\mu} \frac{m_{B}}{n \cdot p-m_{B}}\left[\frac{n \cdot p}{m_{B}} f_{B \pi}^{+}\left(q^{2}\right)-f_{B \pi}^{0}\left(q^{2}\right)\right]\right\} \\
& \quad+\int_{\omega_{s}}^{+\infty} d \omega^{\prime} \frac{1}{\omega^{\prime}-\bar{n} \cdot p-i 0}\left[\rho^{h}\left(\omega^{\prime}, n \cdot p\right) n_{\mu}+\tilde{\rho}^{h}\left(\omega^{\prime}, n \cdot p\right) \bar{n}_{\mu}\right],
\end{aligned}
$$

where the threshold parameter $\omega_{s}$ in the pion channel scales as $\Lambda^{2} / m_{b}$. Matching the hadronic and OPE representations of the vacuum-to- $B$-meson correlation function leads to

$$
\begin{aligned}
f_{B \pi}^{+}\left(q^{2}\right) & =\frac{\tilde{f}_{B}(\mu) m_{B}}{f_{\pi} n \cdot p} \exp \left[\frac{m_{\pi}^{2}}{n \cdot p \omega_{M}}\right] \int_{0}^{\omega_{s}} d \omega^{\prime} e^{-\omega^{\prime} / \omega_{M}} \phi_{B}^{-}\left(\omega^{\prime}\right)+O\left(\alpha_{s}\right), \\
f_{B \pi}^{0}\left(q^{2}\right) & =\frac{n \cdot p}{m_{B}} f_{B \pi}^{+}\left(q^{2}\right)+O\left(\alpha_{s}\right) .
\end{aligned}
$$

Now we are in a position to demonstrate QCD factorization for $\Pi_{\mu}(n \cdot p, \bar{n} \cdot p)$ at one loop using the diagrammatical approach. Technically, this amounts to justifying the equivalence between the soft 


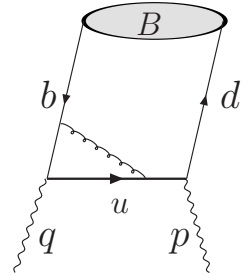

(a)

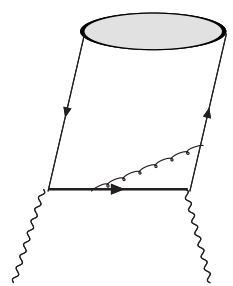

(b)

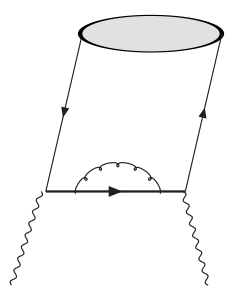

(c)

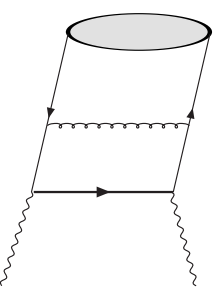

(d)

Figure 1. Next-to-leading-order QCD correction to the correlation function $\Pi_{\mu}(n \cdot p, \bar{n} \cdot p)$.

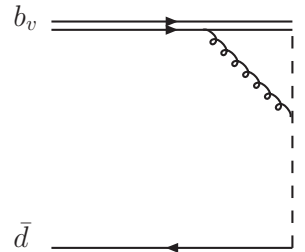

(a)

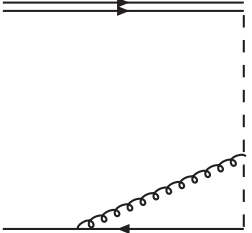

(b)

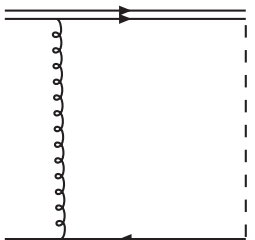

(c)

Figure 2. One-loop effective diagrams for the infrared subtraction.

contribution to the one-loop QCD diagrams displayed in Figure 1 and the infrared subtraction shown in Figure 2 in the heavy quark limit and extracting the hard and hard-collinear contributions to the correlation function at leading power in $\Lambda / m_{b}$. We will apply the standard strategies to establish the diagrammatical factorization for QCD Green functions at one-loop accuracy [13, 14]:

- Identify the leading regions of the QCD amplitudes with the power counting scheme (2);

- Evaluate the loop integrals with the method of regions and extract the "bare" perturbative kernels;

- Apply the ultraviolet renormalization and perform the infrared subtraction;

- Substitute the momentum-space light-cone projector of the $B$-meson.

Taking the weak vertex diagram displayed in Figure 1(a) as an example, the corresponding QCD amplitude can be written as [12]

$$
\begin{aligned}
\Pi_{\mu, \text { weak }}^{(1)=} & \frac{g_{s}^{2} C_{F}}{2(\bar{n} \cdot p-\omega)} \int \frac{d^{D} l}{(2 \pi)^{D}} \frac{1}{\left[(p-k+l)^{2}+i 0\right]\left[\left(m_{b} v+l\right)^{2}-m_{b}^{2}+i 0\right]\left[l^{2}+i 0\right]} \\
& \bar{d}(k) / n \gamma_{5} \hbar \gamma_{\rho}(p-k+\not) \gamma_{\mu}\left(m_{b} / v+\not+m_{b}\right) \gamma^{\rho} b(v) .
\end{aligned}
$$

Employing the power counting scheme (2) one can readily identify that the leading power contributions to $\Pi_{\mu \text {,weak }}^{(1)}$ come from the hard, hard-collinear and soft regions. Expanding the QCD amplitude $\Pi_{\mu, \text { weak }}^{(1)}$ in terms of the powers of $\Lambda / m_{b}$ in the soft region and keeping the leading-order terms yield

$$
\begin{aligned}
\Pi_{\mu, \text { weak }}^{(1), s}= & \frac{g_{s}^{2} C_{F}}{2(\bar{n} \cdot p-\omega)} \int \frac{d^{D} l}{(2 \pi)^{D}} \frac{1}{[\bar{n} \cdot(p-k+l)+i 0][v \cdot l+i 0]\left[l^{2}+i 0\right]} \\
& \bar{d}(k) \ln \gamma_{5} / \bar{n} \gamma_{\mu} b(v) .
\end{aligned}
$$


On the other hand, the one-loop correction to the $B$-meson DA can be computed with the Wilson-line Feynman rules

$$
\begin{aligned}
\Phi_{b \bar{d}, a}^{\alpha \beta,(1)}\left(\omega, \omega^{\prime}\right)= & i g_{s}^{2} C_{F} \int \frac{d^{D} l}{(2 \pi)^{D}} \frac{1}{[\bar{n} \cdot l+i 0][v \cdot l+i 0]\left[l^{2}+i 0\right]} \\
& \times\left[\delta\left(\omega^{\prime}-\omega-\bar{n} \cdot l\right)-\delta\left(\omega^{\prime}-\omega\right)\right][\bar{d}(k)]_{\alpha}[b(v)]_{\beta},
\end{aligned}
$$

from which the infrared subtraction term can be deduced as $\Phi_{b \bar{d}, a}^{(1)} \otimes T^{(0)}=\Pi_{\mu, \text { weak }}^{(1), ~}$, verifying QCD factorization for the vacuum-to- $B$-meson correlation function at one loop diagrammatically. By proceeding in a similar way, the hard-colliner contribution from the weak vertex diagram can be extracted as follows:

$$
\begin{aligned}
\Pi_{\mu, \text { weak }}^{(1), h c}= & \frac{g_{s}^{2} C_{F}}{2(\bar{n} \cdot p-\omega)} \int \frac{d^{D} l}{(2 \pi)^{D}} \frac{2 m_{b} n \cdot(p+l)}{\left[n \cdot(p+l) \bar{n} \cdot(p-k+l)+l_{\perp}^{2}+i 0\right]\left[m_{b} n \cdot l+i 0\right]\left[l^{2}+i 0\right]} \\
& \bar{d}(k) \ln \gamma_{5} \text { 公 } \gamma_{\mu} b\left(p_{b}\right) .
\end{aligned}
$$

which can be further computed with the loop integrals collected in the Appendix A of [12]. Finally, expanding the QCD amplitude (8) in the hard region gives rise to

$$
\begin{aligned}
\Pi_{\mu, \text { weak }}^{(1), h}= & \frac{\alpha_{s} C_{F}}{4 \pi} \tilde{f}_{B}(\mu) m_{B} \frac{\phi_{b \bar{d}}^{-}(\omega)}{\bar{n} \cdot p-\omega}\left\{\overline { n } _ { \mu } \left[\frac{1}{\epsilon^{2}}+\frac{1}{\epsilon}\left(2 \ln \frac{\mu}{n \cdot p}+1\right)+2 \ln ^{2} \frac{\mu}{n \cdot p}+2 \ln \frac{\mu}{m_{b}}\right.\right. \\
& \left.\left.-\ln ^{2} r-2 \operatorname{Li}_{2}\left(-\frac{\bar{r}}{r}\right)+\frac{2-r}{r-1} \ln r+\frac{\pi^{2}}{12}+3\right]+n_{\mu}\left[\frac{1}{r-1}\left(1+\frac{r}{\bar{r}} \ln r\right)\right]\right\},
\end{aligned}
$$

with $r=n \cdot p / m_{b}$ and $\bar{r}=1-r$.

Along the same vein, one can evaluate the leading power contributions to the remaining diagrams in Figure 1 and the resulting factorization formulae for the correlation function are given by

$$
\begin{aligned}
& \Pi=\tilde{f}_{B}(\mu) m_{B} \sum_{k= \pm} C^{(k)}(n \cdot p, \mu) \int_{0}^{\infty} \frac{d \omega}{\omega-\bar{n} \cdot p} J^{(k)}\left(\frac{\mu^{2}}{n \cdot p \omega}, \frac{\omega}{\bar{n} \cdot p}\right) \phi_{B}^{(k)}(\omega, \mu), \\
& \widetilde{\Pi}=\tilde{f}_{B}(\mu) m_{B} \sum_{k= \pm} \widetilde{C}^{(k)}(n \cdot p, \mu) \int_{0}^{\infty} \frac{d \omega}{\omega-\bar{n} \cdot p} \widetilde{J}^{(k)}\left(\frac{\mu^{2}}{n \cdot p \omega}, \frac{\omega}{\bar{n} \cdot p}\right) \phi_{B}^{(k)}(\omega, \mu),
\end{aligned}
$$

where the hard and hard-collinear functions at one loop read [12]

$$
\begin{aligned}
& C^{(+)}=\tilde{C}^{(+)}=1, \quad C^{(-)}=\frac{\alpha_{s} C_{F}}{4 \pi} \frac{1}{\bar{r}}\left[\frac{r}{\bar{r}} \ln r+1\right], \\
& \tilde{C}^{(-)}=1-\frac{\alpha_{s} C_{F}}{4 \pi}\left[2 \ln ^{2} \frac{\mu}{n \cdot p}+5 \ln \frac{\mu}{m_{b}}-\ln ^{2} r-2 \mathrm{Li}_{2}\left(-\frac{\bar{r}}{r}\right)+\frac{2-r}{r-1} \ln r+\frac{\pi^{2}}{12}+5\right],
\end{aligned}
$$

and

$$
\begin{aligned}
J^{(+)}= & \frac{1}{r} \tilde{J}^{(+)}=\frac{\alpha_{s} C_{F}}{4 \pi}\left(1-\frac{\bar{n} \cdot p}{\omega}\right) \ln \left(1-\frac{\omega}{\bar{n} \cdot p}\right), \quad J^{(-)}=1 \\
\tilde{J}^{(-)}= & 1+\frac{\alpha_{s} C_{F}}{4 \pi}\left[\ln ^{2} \frac{\mu^{2}}{n \cdot p(\omega-\bar{n} \cdot p)}-2 \ln \frac{\bar{n} \cdot p-\omega}{\bar{n} \cdot p} \ln \frac{\mu^{2}}{n \cdot p(\omega-\bar{n} \cdot p)}\right. \\
& \left.-\ln ^{2} \frac{\bar{n} \cdot p-\omega}{\bar{n} \cdot p}-\left(1+\frac{2 \bar{n} \cdot p}{\omega}\right) \ln \frac{\bar{n} \cdot p-\omega}{\bar{n} \cdot p}-\frac{\pi^{2}}{6}-1\right] .
\end{aligned}
$$



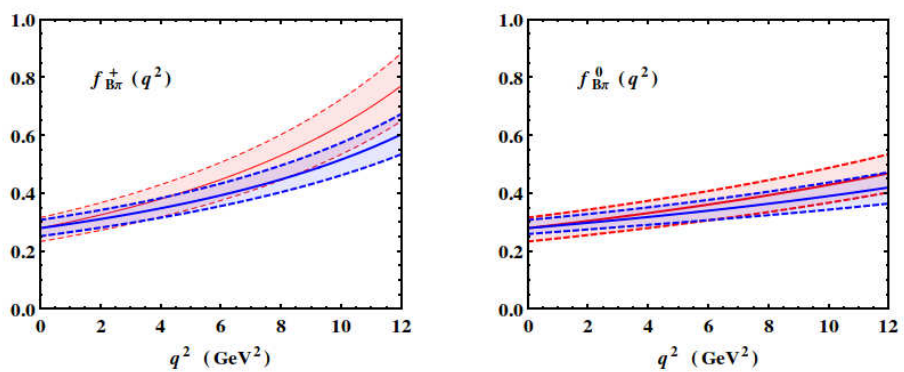

Figure 3. The momentum-transfer dependence of the $B \rightarrow \pi$ form factors $f_{B \pi}^{+, 0}\left(q^{2}\right)$ from the NLL resummation improved LCSR (17) with the $B$-meson DA (pink curves) and from the NLO QCD sum rules with the pion DA (blue curves).

The hard coefficients presented in (14) are in compatible with the perturbative matching coefficients of the weak current $\bar{q} \gamma_{\mu} b$ from QCD to soft-collinear effective theory (SCET) [15], and the hardcollinear functions displayed in (15) coincide with the corresponding jet functions computed with the SCET Feynman rules [11].

It is evident that there is no common value of $\mu$ that can avoid the parametrically large logarithms of order $\ln \left(m_{b} / \Lambda\right)$ in the hard functions, the jet functions and the $B$-meson DA. The summation of these large logarithms in the hard coefficient functions can be accomplished by solving the following renormalization-group equations

$$
\begin{aligned}
\frac{d}{d \ln \mu} \tilde{C}^{(-)}(n \cdot p, \mu) & =\left[-\Gamma_{\text {cusp }}\left(\alpha_{s}\right) \ln \frac{\mu}{n \cdot p}+\gamma\left(\alpha_{s}\right)\right] \tilde{C}^{(-)}(n \cdot p, \mu), \\
\frac{d}{d \ln \mu} \tilde{f}_{B}(\mu) & =\tilde{\gamma}\left(\alpha_{s}\right) \tilde{f}_{B}(\mu),
\end{aligned}
$$

where the three-loop cusp anomalous dimension and the two-loop $\gamma(\tilde{\gamma})$ are needed to achieve the nextto-leading-logarithmic (NLL) resummation. Applying the standard procedure for the construction of QCD sum rules leads to

$$
\begin{aligned}
& f_{\pi} e^{-m_{\pi}^{2} /\left(n \cdot p \omega_{M}\right)}\left\{\frac{n \cdot p}{m_{B}} f_{B \pi}^{+}\left(q^{2}\right), f_{B \pi}^{0}\left(q^{2}\right)\right\} \\
& =\left[U_{2}\left(\mu_{h 2}, \mu\right) \tilde{f}_{B}\left(\mu_{h 2}\right)\right] \int_{0}^{\omega_{s}} d \omega^{\prime} e^{-\omega^{\prime} / \omega_{M}}\left[\left[U_{1}\left(n \cdot p, \mu_{h 1}, \mu\right) \widetilde{C}^{(-)}\left(n \cdot p, \mu_{h 1}\right)\right] \phi_{B, \mathrm{eff}}^{-}\left(\omega^{\prime}, \mu\right)\right. \\
& \left.\quad+r \phi_{B, \mathrm{eff}}^{+}\left(\omega^{\prime}, \mu\right) \pm \frac{n \cdot p-m_{B}}{m_{B}}\left(\phi_{B, \mathrm{eff}}^{+}\left(\omega^{\prime}, \mu\right)+C^{(-)}(n \cdot p, \mu) \phi_{B}^{-}\left(\omega^{\prime}, \mu\right)\right)\right]
\end{aligned}
$$

where the explicit expressions of $\phi_{B, \text { eff }}^{ \pm}\left(\omega^{\prime}, \mu\right)$ can be found in [12].

Having at our disposal the NLL resummation improved LCSR presented in (17), it is straightforward to plot the $q^{2}$ dependence of the $B \rightarrow \pi$ form factors $f_{B \pi}^{+, 0}\left(q^{2}\right)$ in Figure 3 where the theoretical predictions from the sum rules with the pion DA [4] are also shown for a comparison. The observed discrepancy of the $q^{2}$ shape for the vector form factor $f_{B \pi}^{+}\left(q^{2}\right)$ predicted from the two distinct sum rules could be attributed to the systematical uncertainties generated by the different parton-hadron duality 
ansatz and to the yet unaccounted higher order/power corrections in the perturbative calculations of the corresponding correlation functions. Expressing the differential branching fraction of $B \rightarrow \pi \mu v_{\mu}$ in terms of the form factor $f_{B \pi}^{+}\left(q^{2}\right)$

$$
\frac{d \Gamma}{d q^{2}}\left(B \rightarrow \pi \mu v_{\mu}\right)=\frac{G_{F}^{2}\left|V_{u b}\right|^{2}}{24 \pi^{3}}\left|\vec{p}_{\pi}\right|^{3}\left|f_{B \pi}^{+}\left(q^{2}\right)\right|^{2} .
$$

and employing the experimental measurements for the integrated decay rate $[16,17]$ lead to

$$
\left|V_{u b}\right|=\left(\left.3.05_{-0.38}^{+0.54}\right|_{\text {th. }} \pm\left. 0.09\right|_{\text {exp. }}\right) \times 10^{-3},
$$

which is consistent with the exclusive determination of $\left|V_{u b}\right|$ from the leptonic $B \rightarrow \tau v_{\tau}$ decay [18].

\section{Conclusion}

To summarize, we demonstrated QCD factorization for the vacuum-to- $B$-meson correlation function used in the construction of the $B \rightarrow \pi$ form factors at one-loop accuracy explicitly with the method of regions and achieved the resummation of large logarithms in the short-distance functions at NLL by solving the renormalization-group equations in the momentum space. Phenomenological applications of the newly derived sum rules with the $B$-meson DA were also discussed in brief, focusing on the extraction of exclusive $\left|V_{u b}\right|$ from the semi-leptonic $B \rightarrow \pi \mu v_{\mu}$ decay.

\section{References}

[1] M. Beneke and T. Feldmann, Nucl. Phys. B 592 (2001) 3 [hep-ph/0008255].

[2] P. Ball and R. Zwicky, Phys. Rev. D 71, 014015 (2005) [hep-ph/0406232].

[3] G. Duplancic, A. Khodjamirian, T. Mannel, B. Melic and N. Offen, JHEP 0804, 014 (2008) [arXiv:0801.1796 [hep-ph]].

[4] A. Khodjamirian, T. Mannel, N. Offen and Y.-M. Wang, Phys. Rev. D 83, 094031 (2011) [arXiv:1103.2655 [hep-ph]].

[5] Y. M. Wang, Y. Li and C. D. Lü, Eur. Phys. J. C 59, 861 (2009) [arXiv:0804.0648 [hep-ph]].

[6] H. n. Li, Y. L. Shen and Y. M. Wang, Phys. Rev. D 85, 074004 (2012) [arXiv:1201.5066 [hep-ph]].

[7] C. D. Lü, Y. M. Wang, H. Zou, A. Ali and G. Kramer, Phys. Rev. D 80, 034011 (2009) [arXiv:0906.1479 [hep-ph]].

[8] A. Khodjamirian, T. Mannel and N. Offen, Phys. Lett. B 620, 52 (2005) [hep-ph/0504091].

[9] A. Khodjamirian, T. Mannel and N. Offen, Phys. Rev. D 75, 054013 (2007) [hep-ph/0611193].

[10] F. De Fazio, T. Feldmann and T. Hurth, Nucl. Phys. B 733, 1 (2006) [hep-ph/0504088].

[11] F. De Fazio, T. Feldmann and T. Hurth, JHEP 0802, 031 (2008) [arXiv:0711.3999 [hep-ph]].

[12] Y. M. Wang and Y. L. Shen, Nucl. Phys. B 898, 563 (2015) [arXiv:1506.00667 [hep-ph]].

[13] Y. M. Wang and Y. L. Shen, JHEP 1602, 179 (2016) [arXiv:1511.09036 [hep-ph]].

[14] Y. M. Wang, arXiv:1606.03080 [hep-ph].

[15] C. W. Bauer, S. Fleming, D. Pirjol and I. W. Stewart, Phys. Rev. D 63, 114020 (2001) [hep$\mathrm{ph} / 0011336]$.

[16] J. P. Lees et al. [BaBar Collaboration], Phys. Rev. D 86, 092004 (2012) [arXiv:1208.1253 [hepex]].

[17] A. Sibidanov et al. [Belle Collaboration], Phys. Rev. D 88, 032005 (2013) [arXiv:1306.2781 [hep-ex]].

[18] B. Kronenbitter et al. [Belle Collaboration], Phys. Rev. D 92, 051102 (2015) [arXiv:1503.05613 [hep-ex]]. 